\title{
When New becomes Old
}

\author{
Alessandro Ederoclite* \\ Centro de Estudios de Física del Cosmos de Aragón \\ E-mail: aederoclecefca.es
}

\section{Claus Tappert}

Universidad de Valparaiso

\section{Linda Schmitdobreick}

European Southern Observatory

\section{Nikolaus Vogt , Irma Fuentes Morales}

Universidad de Valparaiso

\section{Héctor Vázquez Ramió, Javier Abril-Ibañez}

Centro de Estudios de Física del Cosmos de Aragón

Classical novae have been studied for over a century but the relation between these explosions and their host systems is still far from complete. In this talk, I review a project aimed at building a statistically significant sample of old nova systems and I analyse the role of future narrow-band surveys in the search for these objects.

The Golden Age of Cataclysmic Variables and Related Objects - III, Golden2015

7-12 September 2015

Palermo, Italy

\footnotetext{
*Speaker.
} 


\section{Introduction}

Classical Novae $(\mathrm{CNe})$ are thermonuclear explosions which occur on the surface of a white dwarf which is accreting mass from a less evolved companion.

The "Old Nova Project" started 5 years ago with the idea to make a complete status of the properties of the binaries which harboured nova explosions. The fundamental questions which drove the beginning of the project are: (i) how does the population of old novae look like (ii) how does the population of old novae compare to CVs? (iii) do short-period old novae exist in a significant number? (iv) what is the role of magnetic fields on the $\mathrm{CN}$-explosion? In order to make sure that the spectrum was not dominated by the remnant, we established that the nova explosion had to had occurred, at least, 30 years before the observations. Given the nature of such a constraint, at the beginning of our project, we had a sample of 204 novae (novae exploded before 1980, see [7],[8]), while now the sample has increased to 223 (novae exploded before 1986).

Our strategy can be summarised in three main steps. If the old nova is not known and/or the coordinates of the nova are not precise enough to identify the object, we take $U B V R$ photometry of the region. We then perform spectroscopy of the candidates which we selected as not lying in the region where all "normal" stars are located. We also take spectra of these novae whose position is known. Finally, if the object is bright enough, we also proceed to time-resolved photometry or spectroscopy for period determination. For more details, see [8]. The results are being given in a series of papers ([17], [18], [19], [20], [21]).

\section{Novae in the Northern Hemisphere}

A variety of projects have observed old novae in the Northern hemisphere (here we follow the convention of our group where the Northern hemisphere is for $\delta>-20$ degrees and the Southern hemisphere has $\delta<20$ degrees, therefore having an overlap around the celestial equator, see [7] and [8]). Out of the 114 Northern novae, according to [5], the period is unknown for 87 objects and for 30 objects there is not even a candidate for the old nova.

Most of the information on old novae in the northern hemisphere come from dedicated projects (e.g. [15] or [14]). In some other cases, old novae can be found as by-product of surveys with different main science goals, like SDSS ([16]), IPHAS ([1]) and UVEX ([22]). Despite these works, the number of old novae without confirmation (and/or without period determination) is disturbingly high.

The search for CVs in a survey like SDSS introduces a series of selection effects which are well described in [10]. This is due to the fact that SDSS is aimed at quasars and CVs are a by-product of the main survey.

\section{Future Narrow-band Surveys}

The Observatorio Astrofísico de Javalambre (OAJ; see [3] and [4]) is a new astronomical facility located in mainland Spain at an altitude of $1957 \mathrm{~m}$ above sea level and a median seeing of $\sim 0.7$ " [11]. 
Two main telescopes are hosted at OAJ: the "Javalambre Survey Telescope" (JST) and the "Javalambre Auxiliary Survey Telescope". The JST is a 2.5m Ritchey-Chrétien telescope, with a field corrector which provides optical quality over a full 3sq.deg. field of view. The JAST is an $80 \mathrm{~cm}$ telescope (also Ritchey-Chrétien), with a 2sq.deg. field of view.

The JAST has been designed to provide the photometric calibration for the largest telescope. The instrument which will be installed on JAST is T80Cam. This is a camera equipped with a $9 \mathrm{k} \times 9 \mathrm{k}$ CCD and 12 filters, four SDSS (griz), one modification of the Sloan $u$ and seven narrow band filters, especially selected for the characterization of stellar spectra (see Fig.1).

The JST has been designed to perform the J-PAS survey [2]. It is equipped with JPCam, a panoramic camera, with $149 \mathrm{k} \times 9 \mathrm{k}$ CCDs. JPCam has five filter trays, each carrying 14 filters, so that each CCD observes through a filter. An easy way to understand how JPCam works is to think about it as 14 contiguous and independent cameras observing adjacent sky regions. The filter set

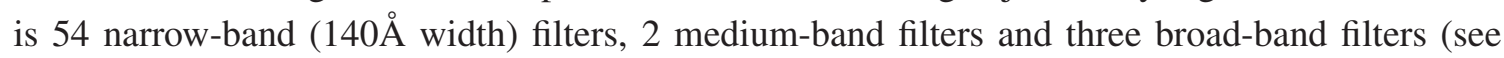
Fig.1).
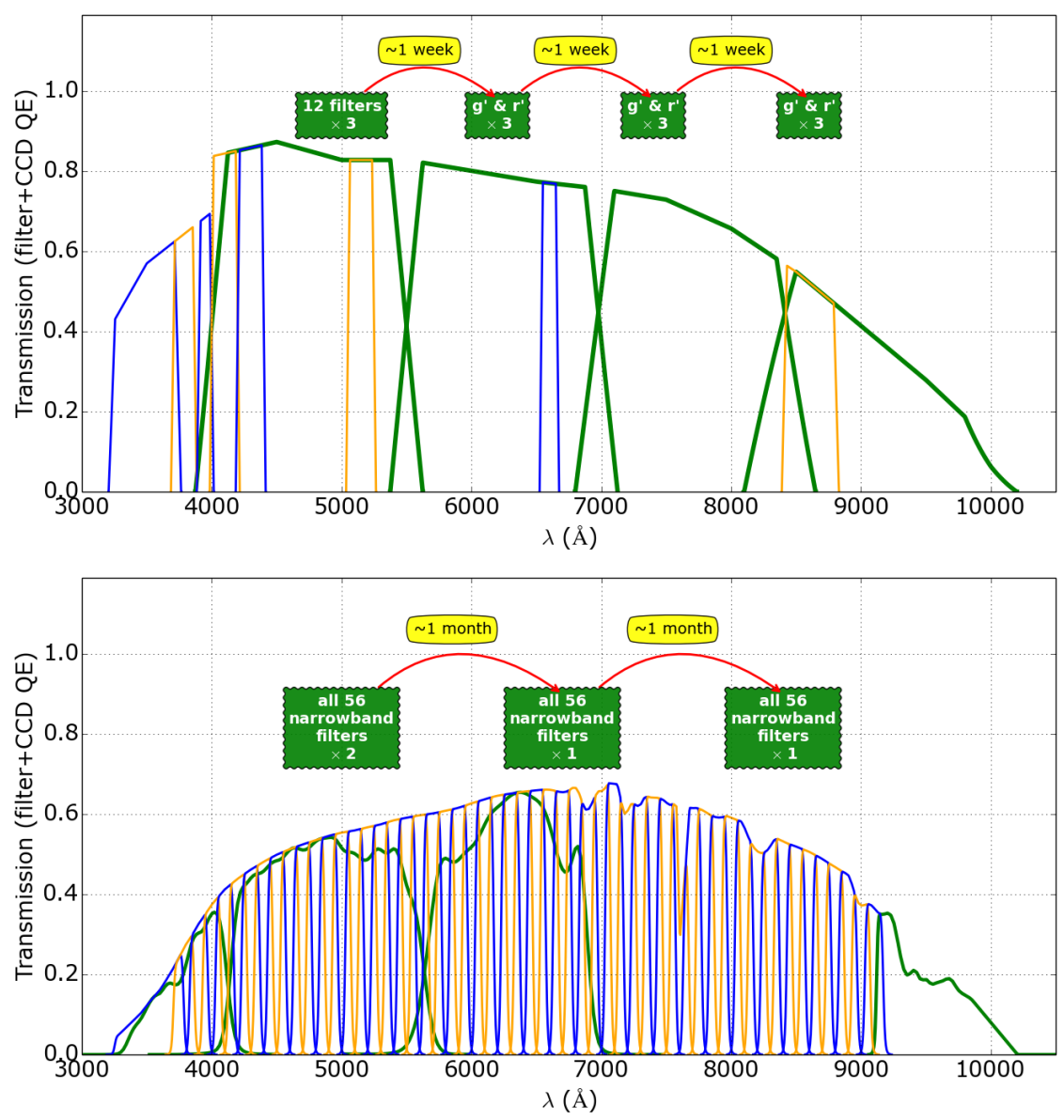

Figure 1: The upper panel shows the response curves of the filters installed on T80Cam as well as the observing strategy which will be followed for each pointing of the J-PLUS survey. The lower panel shows the same but for JPCam and J-PAS. 
Expected to start in early 2017, J-PAS has been designed to be a cosmological survey and to get narrow-band photometry for 8500sq.deg. of the sky down to $\operatorname{mag}_{\mathrm{AB}} \sim 22.5-23.5$ (depending on the filter). J-PLUS, the survey being carried out with the T80 (and expected to start by the end of 2015), will cover the same area. Although, in principle, J-PLUS is designed to perform a photometric calibration, it has its own scientific potential. Fig. 2 shows how the SED of a CV will look once observed with both J-PLUS and J-PAS.

[9] explains extensively how CVs are recovered in big surveys and the problem of telling CVs from quasars. The left panel of Fig.3 shows how CVs (bottom two panels) can be easily differenciated from "normal" stars (second and third panel) using a combination of narrow band filters, while quasars (top panel) can be problematic in some specific redshift ranges (e.g. the right panel of the same figure). Indeed, in Fig.4, one can see how CVs and quasars overlap using the same colour-magnitude and colour-colour diagrams used in [6] comparing SDSS and CRTS. Even a combination of the two most promising colours from these two works is not completely satisfactory (bottom-right panel). The combination of filters centred at $4100 \AA$, $6600 \AA$, and $8610 \AA$ (with the respective broad band filters to provide the continua) provided by J-PLUS, is going to offer the tool to separate CVs from quasars in ranges like $0.3<z<0.4$, where the use of $\mathrm{H}_{\alpha}$ excess is insufficient. Interestingly, in this redshift range, about one quasar per square degree is foreseen to be observed by J-PLUS (Bonoli, private communication) while, based on [12] and [13] ,[2] expect to detect at least $0.5 \mathrm{CV}$ s per square degree.

\section{Summary and Conclusions}

In the last five years, we have undertaken the effort to recover and characterise novae which have exploded more than 30 years ago. The project has been quite successful by now (see [23] and [24]).

In order to improve the discovery efficiency in the Northern hemisphere, we are planning on taking advantage from a new observing facility, the Observatorio Astrofísico de Javalambre, where two wide-field surveys will be carried out. In particular, the first project (J-PLUS) will provide soon 8500sq.deg. of sky observed in broad and narrow-band filters which appear ideal to distinguish CVs (and old novae in particular) from other astrophysical sources.

\section{Acknowledgments}

This research has been supported by the project AYA2012-30789, co-funded by the Spanish MINECO and European Funds. Moreover, CT, IFM and NV acknowledge support from the FONDECYT Regular grant 1120338 and the Centro de Astrofísica de Valparaíso.

\section{References}

[1] Barentsen, G. et al. The second data release of the INT Photometric $H_{\alpha}$ Survey of the Northern Galactic Plane (IPHAS DR2) 2014, MNRAS, 444, 3230

[2] N. Benitez et al. J-PAS: The Javalambre-Physics of the Accelerated Universe Astrophysical Survey 2014, [astro-ph/1403.5237] 


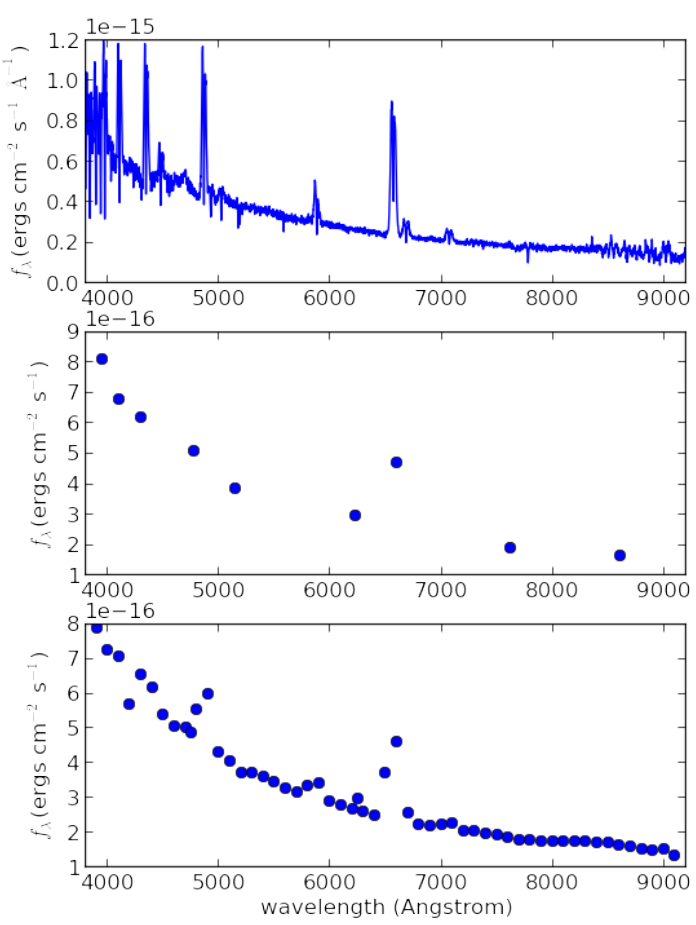

Figure 2: From top to bottom: the SDSS spectrum of NZ Boo and the SED of the same object obtained convolving the SDSS spectrum with the J-PLUS and J-PAS filter set, respectively.

[3] The Observatorio Astrofísico de Javalambre: goals and current status 2012, SPIE, 8448, 1

[4] The Observatorio Astrofísico de Javalambre: current status, developments, operations, and strategies 2014, SPIE, 9149, 1

[5] Downes, R. et al. A Catalog and Atlas of Cataclysmic Variables: The Final Edition 2005, JAD, 11, 2

[6] Drake, A.J. et al. Cataclysmic variables from the Catalina Real-time Transient Survey 2014, ApJS, 213,9

[7] Ederoclite et al. Old Novae: When New Becomes Old 2011, ASPC, 447, 215

[8] Ederoclite et al. When a Nova Becomes Old 2013, AcPPP, 2, 226

[9] Gaensicke, B. T. Observational population studies of cataclysmic variables - The golden era of surveys 2005, ASPC, 330, 3

[10] Gaensicke, B. et al. SDSS unveils a population of intrinsically faint cataclysmic variables at the minimum orbital period 2009, MNRAS, 397, 2170

[11] Moles, M. et al. Site Testing of the Sierra de Javalambre: First Results 2010, PASP, 122, 363

[12] Ortiz, R. \& Lepine, J.R. D. A model of the Galaxy for predicting star counts in the infrared 1993, A\&A, 279, 90

[13] Patterson, J. Late Evolution of Cataclysmic Variables 1998, PASP, 110, 1132 

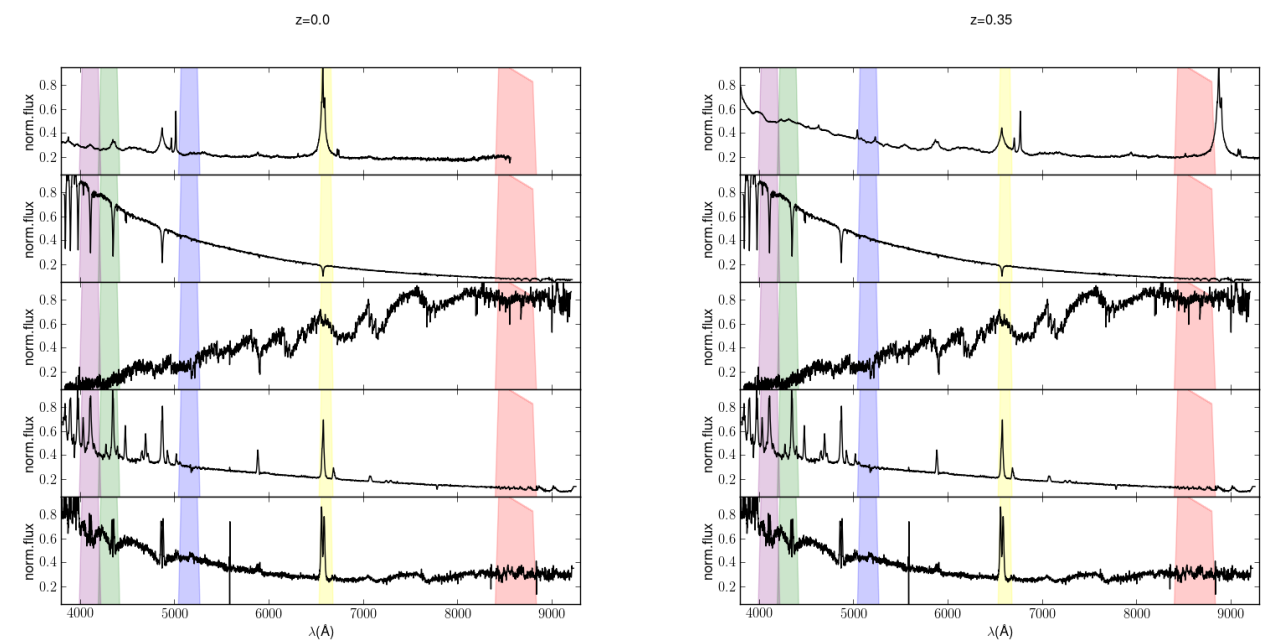

Figure 3: The left panel shows, from top to bottom, a quasar template at $z=0.0$, a hot star spectrum (one of the templates of SDSS-DR4), a cool star spectrum (again, one of the templates of SDSS-DR4), the spectrum of a high mass-transfer $\mathrm{CV}$ and the spectrum of a low mass-transfer $\mathrm{CV}$. The right panel shows the same as the second panel but the quasar has been moved to $z=0.35$. Note how, at this redshift the $\mathrm{H} \beta$ line of the quasar can be confused with the $\mathrm{H}_{\alpha}$ line of a CV. The plot is intended to show the position of spectral features with respect to the J-PLUS narrow-band filters, therefore, for clarity, the spectra are normalised to their maximum value in the optical wavelength range.

[14] Ringwald, F. A., Naylor, T. and Mukai, K. The optical spectra of old novae 1996, MNRAS, 281, 192

[15] Szkody, P. \& Howell, S. B. Spectroscopic confirmation of high galactic latitude cataclysmic variables 1992, ApJ, 78, 537

[16] Szkody et al. Cataclysmic Variables from the Sloan Digital Sky Survey. VIII. The Final Year (2007-2008) 2011, AJ, 142, 181

[17] Tappert, C. et al. Life after eruption - I. Spectroscopic observations of 10 nova candidates 2012, MNRAS, 423, 2476

[18] Tappert, C. et al. Life after eruption - II. The eclipsing old nova V728 Scorpii 2013, MNRAS, 431, 92

[19] Tappert, C. et al. Life after eruption - III. Orbital periods of the old novae V365 Car, AR Cir, V972 Oph, HS Pup, V909 Sgr, V373 Sct and CN Vel 2013, MNRAS, 436, 2412

[20] Tappert, C. et al. Life after eruption - IV. Spectroscopy of 13 old novae 2014, MNRAS, 442, 565

[21] Tappert, C. et al. Life after eruption - V. Spectroscopy of eight candidate old novae with Gemini-South 2015, MNRAS, 450, 943

[22] Verbeek, K. et al. A first catalogue of automatically selected ultraviolet-excess sources from the UVEX survey 2012, MNRAS, 420, 1115

[23] Tappert, C. et al. 2015, this workshop

[24] Fuentes Morales, I. et al. 2015, this workshop 

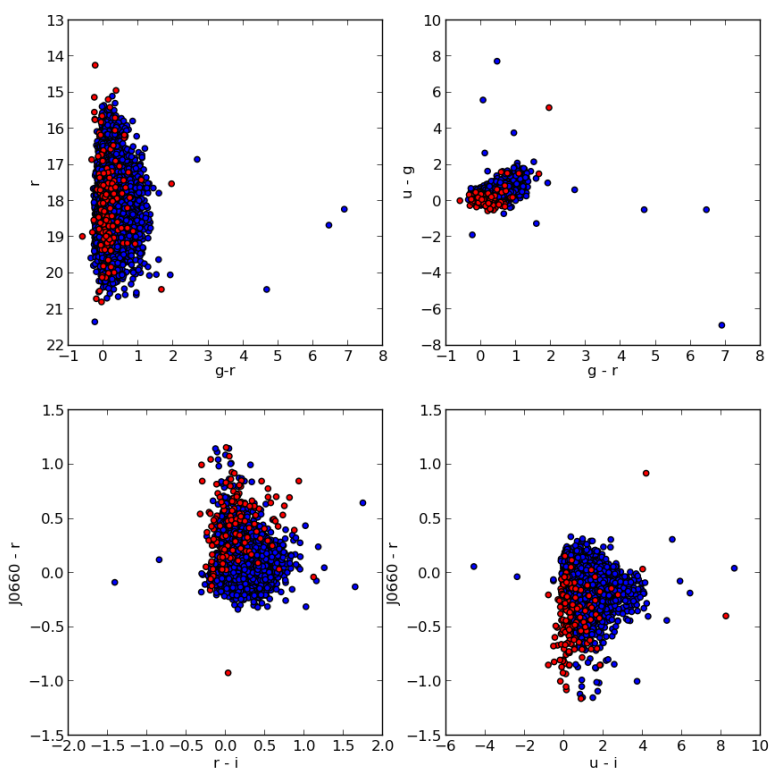

Figure 4: Red points are SDSS spectra of CVs (from [10]) convolved with J-PLUS filters. Blue points are quasars. When the convolution was not possible (e.g. $u$ filter), we used SDSS-DR10 photometry. The upper-left panel shows a colour-magnitude diagram $r$ vs. $g-r$, the upper-right panel shows a colour-colour diagram $u-g$ vs. $g-r$, following [6]. The bottom-left panel shows a colour-colour diagram $\left(\mathrm{H}_{\alpha}-r\right.$ vs. $\left.r-i\right)$ following [1]. Finally, the bottom-right panel shows a colour-colour diagram using the two most promising colours from the previous works: $u-g$ and $\mathrm{H}_{\alpha}-r$.

\section{DISCUSSION}

DAVID BUCKLEY: What is the observation time required for all the 59 filters and the limiting magnitude.

ALESSANDRO EDEROCLITE: The limiting magnitude of J-PAS (the survey to be carried out with the JST/T250) is 22.5 or 23, depending on the filter. Each field is going to be observed with the 59 filters but not in the same night. For a whole description of the straetgy survey, please, refer to $[2]$ 